\title{
Entanglement and decoherence: fragile and robust entanglement
}

\author{
Jaroslav Novotný, ${ }^{1,2}$ Gernot Alber, ${ }^{2}$ and Igor Jex ${ }^{1}$ \\ ${ }^{1}$ Department of Physics, FNSPE, Czech Technical University in Prague, \\ Břehová 7, 11519 Praha 1 - Staré Město, Czech Republic \\ ${ }^{2}$ Institut für Angewandte Physik, Technische Universität Darmstadt, D-64289 Darmstadt, Germany
}

\begin{abstract}
The destruction of entanglement of open quantum systems by decoherence is investigated in the asymptotic long-time limit. Starting from a general and analytically solvable decoherence model which does not involve any weak-coupling or Markovian assumption it is shown that two fundamentally different classes of entangled states can be distinguished. Quantum states of the first class are fragile against decoherence so that they can be disentangled asymptotically even if coherences between pointer states are still present. Quantum states of the second type are robust against decoherence. Asymptotically they can be disentangled only if also decoherence is perfect. A simple criterion for identifying these two classes on the basis of two-qubit entanglement is presented.
\end{abstract}

PACS numbers: 03.67.Mn,03.65.Ud,03.65.Yz

Every natural physical quantum object is in contact with an environment. A consequence of the resulting inevitable interaction of such an open quantum system with its environment is decoherence [1] 3$]$. For sufficiently long interaction times this process leads to an environmentinduced selection of so-called pointer states which remain at least approximately undisturbed while any of their superposition states decays quickly into a mixture. This asymptotic loss of quantum coherence is responsible for the appearance of classical features of open quantum systems [1 3]. Apart from that decoherence is also a process which tends to destroy entanglement. Nevertheless, the recently discussed time-dependent phenomenon of sudden death of entanglement [4] has already hinted at some subtle relations between decoherence and disentanglement in the time domain. Thereby, it has been observed that entanglement of an open quantum system may already disappear after a finite interaction time with its environment while complete decoherence requires an infinite amount of time. A further exploration of the subtleties between entanglement and decoherence is not only of fundamental physical interest but is also of significance for practical applications in the area of quantum information science [5] in which entanglement is a key resource. For the realization of large quantum information processors, for example, it is of vital importance to understand how decoherence-induced loss of entanglement of an open quantum system scales with the size of an environment in the limit of arbitrarily long interaction times. Under which conditions are already finitely sized environments capable of disentangling open quantum systems completely? Are there classes of entangled quantum states whose properties differ significantly under decoherence? It is a main purpose of this letter to explore these questions.

For this purpose a sufficiently general model of decoherence is needed which is exactly solvable without further simplifying assumptions concerning, e.g., sizes of environments, initially prepared quantum states, interac- tion strengths, or correlation times between system and environment. Of particular interest are generic asymptotic long-time properties of such a general decoherence model which exhibit clearly the intricate interplay between decoherence and destruction of entanglement.

Motivated by the practical significance of elementary distinguishable two-level systems (qubits) for purposes of quantum information processing in the following we present a qubit-based class of such decoherence models. Thereby, we start from the recent observation that decoherence of a single system qubit can be modeled by a sequence of 'controlled- $U$ ' unitary transformations in which the single system qubit acts as a control and the environmental qubits act as targets [6]. This result was obtained under the simplifying assumptions that the environment is formed by an infinite number of qubits, that each qubit is prepared initially in the same state so that all environmental qubits are uncorrelated, that the environmental qubits do not interact among themselves, and that each environmental target qubit interacts with the single system qubit only once. In order to overcome all these restrictive approximations let us consider as a natural generalization $k$ system qubits which interact with $n$ environmental qubits by a sequence of elementary interactions or 'collisions'. All of these 'collisions' are assumed to be well separated in time. In each of them a pair of qubits, i.e. a control qubit $i$ and a target qubit $j$, is selected randomly and a controlled unitary transformation

$$
\hat{U}_{i j}^{(\phi)}=|0\rangle_{i i}\left\langle 0\left|\otimes \hat{I}_{j}+\right| 1\right\rangle_{i i}\langle 1| \otimes \hat{u}_{j}^{(\phi)}
$$

is applied. Thereby, $\hat{I}_{j}$ is the unit operator acting on qubit $j$ and

$$
\hat{u}_{j}^{(\phi)}=\cos \phi\left(|0\rangle_{j j}\langle 0|-| 1\rangle_{j j}\langle 1|\right)+\sin \phi\left(|0\rangle_{j j}\langle 1|+| 1\rangle_{j j}\langle 0|\right)
$$

denotes the unitary one-qubit transformation acting on the target qubit $j$. Such controlled unitary couplings have already been investigated in the context of vari- 
ous decoherence models [1, 3] because they do not affect the computational basis states $|0\rangle_{i}$ and $|1\rangle_{i}$ of the control qubit $i$ and at the same time they can decrease any quantum coherence between these two system states. Within our generalized decoherence model each system qubit $i$ is a possible control qubit for an elementary interaction $\hat{U}_{i j}^{(\phi)}$ with any environmental target qubit $j$. Furthermore, all environmental qubits also interact among themselves. However, in order to guarantee a well defined pointer basis in our decoherence model we assume that the system qubits do not interact among themselves. The interaction pattern characterizing which qubits can be coupled by an elementary interaction $\hat{U}_{i j}^{(\phi)}$ between randomly chosen qubits $i$ and $j$ can be encoded in a convenient way in a weighted and directed interaction graph [7]. Its set of vertices represents the $n+k$ qubits of the total qubit system and the set of directed edges $E$ represents the possible interactions among the qubits. Each of these edges $e=i j \in \mathrm{E}$ is weighted with a probability $p_{e}$ with which qubits $i$ and $j$ are coupled by the unitary two-qubit transformation $\hat{U}_{e}^{(\phi)}$. These probabilities are normalized to unity, i.e. $\sum_{e \in \mathrm{E}} p_{e}=1$. A simple example of such a qubit network is depicted in Fig. 1.

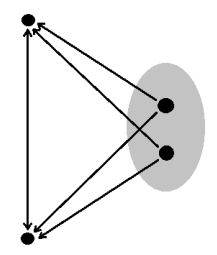

FIG. 1: Schematic representation of a system-environment qubit network: $k=2$ qubits of the open quantum system (grey region) interact with $n=2$ environmental qubits. The directed edges indicate all

possible elementary two-qubit couplings. The tail (head) of an edge symbolizes the control (target) qubit.

Thus, within this decoherence model the quantum state $\hat{\rho}(N)$ resulting from $N$ completed elementary interactions is changed by the $(N+1)$-th elementary interaction to the quantum state

$$
\hat{\rho}(N+1)=\sum_{e \in \mathrm{E}} p_{e} \hat{U}_{e}^{(\phi)} \hat{\rho}(N) \hat{U}_{e}^{(\phi) \dagger} \equiv \mathcal{P}(\hat{\rho}(N)) .
$$

The map $\mathcal{P}$ is a random unitary operation [8] and describes an elementary interaction or 'collision' between system and environment. General properties of quantum states $\hat{\rho}(N)$ resulting from repeated applications of such quantum maps have been studied recently [9]. In particular, it has been shown that in the asymptotic limit of $N \gg 1$ the quantum state $\hat{\rho}(N)$ becomes independent of the probability distribution $\left\{p_{e}, e \in E\right\}$ and that it is determined uniquely by a linear attractor space. This latter space is formed by the maximal set of all possible orthonormal solutions $\hat{X}_{\lambda, i}$ of the eigenvalue equations

$$
\hat{U}_{e}^{(\phi)} \hat{X}_{\lambda, i} \hat{U}_{e}^{(\phi) \dagger}=\lambda \hat{X}_{\lambda, i} \text { for all } e \in \mathrm{E}
$$

with $|\lambda|=1$. The index $i$ distinguishes different mutually orthonormal solutions with eigenvalue $\lambda$. If these attractors are known, in the asymptotic limit $N \gg 1$ the quantum state resulting from an initially prepared state $\hat{\rho}_{\text {in }}$ is given by

$$
\hat{\rho}(N)=\mathcal{P}^{N}\left(\hat{\rho}_{i n}\right)=\sum_{|\lambda|=1, i} \lambda^{N} \operatorname{Tr}\left\{\hat{\rho}_{i n} \hat{X}_{\lambda, i}^{\dagger}\right\} \hat{X}_{\lambda, i}
$$

With the help of the methods developed in Ref. [7] it can be shown that within our decoherence model for $n \geq 2$ the only possible eigenvalue of Eqs. (4) is given by $\lambda=1$ and its eigenspace is spanned by the $4^{k}+3 \times 2^{k}+1$ linear independent solutions

$$
\begin{aligned}
& |\boldsymbol{x}\rangle\left\langle\boldsymbol{x}\left|\otimes \hat{I}_{n},\right| \mathbf{0}\right\rangle\left\langle\boldsymbol{x}|\otimes| \mathbf{0}_{n}\right\rangle\left\langle\phi_{n}|,| \boldsymbol{x}\right\rangle\left\langle\mathbf{0}_{k}|\otimes| \phi_{n}\right\rangle\left\langle\mathbf{0}_{n}\right|, \\
& |\boldsymbol{x}\rangle\left\langle\boldsymbol{y}|\otimes| \phi_{n}\right\rangle\left\langle\phi_{n}|,| \mathbf{0}_{k}\right\rangle\left\langle\mathbf{0}_{k}|\otimes| \mathbf{0}_{n}\right\rangle\left\langle\mathbf{0}_{n}\right|
\end{aligned}
$$

with the pure quantum states $\left|\mathbf{0}_{n}\right\rangle=|0\rangle^{\otimes n},|\phi\rangle=$ $\cos (\phi / 2)|0\rangle+\sin (\phi / 2)|1\rangle,\left|\phi_{n}\right\rangle=|\phi\rangle^{\otimes n}$. Vectors $|\boldsymbol{x}\rangle$ and $|\boldsymbol{y}\rangle$, with $\boldsymbol{x}, \boldsymbol{y} \in 2^{k}$, are arbitrary elements of the computational basis of $k$ qubits. Note that the quantum state $|\phi\rangle$ is not affected by the unitary transformation $\hat{u}^{\phi}$ of Eq.(2), i.e. $\hat{u}^{\phi}|\phi\rangle=|\phi\rangle$.

In order to investigate the decoherence process affecting the $k$ system qubits in the asymptotic limit of large numbers of elementary interactions $N \gg 1$ let us consider an initially prepared quantum state $\hat{\rho}_{i n}=\hat{\rho}^{(S)} \otimes \hat{\rho}^{(E)}$ which does not contain any correlations between system $S$ and environment $E$. Using the attractor space of Eq.(6) and tracing out the environment in Eq.(5) the asymptotic quantum state of the open quantum system $S$ is given by

$$
\hat{\rho}_{\infty}^{(S)}=\operatorname{diag}\left(\hat{\rho}^{(S)}\right)+\sum_{\boldsymbol{x}, \boldsymbol{y} \in 2^{k}, \boldsymbol{x} \neq \boldsymbol{y}}\left\langle\phi_{n}\left|\hat{\rho}^{(E)}\right| \phi_{n}\right\rangle \hat{\rho}_{\boldsymbol{x}, \boldsymbol{y}}^{(S)}|\boldsymbol{x}\rangle\langle\boldsymbol{y}|
$$

with $\operatorname{diag}\left(\hat{\rho}^{(S)}\right)$ denoting the diagonal part of the system state $\hat{\rho}^{(S)}$ with respect to the pointer basis $\left\{|\boldsymbol{x}\rangle, \boldsymbol{x} \in 2^{k}\right\}$. At this point it is worth emphasizing that in view of the general results of Ref. 7] some of the conditions leading to Eq.(7) can be relaxed. Thus, it turns out, for example, that Eq. (7) is already valid if at least one pair of environmental qubits is interacting.

According to Eq.(7) the influence of the environment $E$ on the asymptotic quantum state of the system $S$ is described by the decoherence factor $0 \leq r=\left\langle\phi_{n}\left|\hat{\rho}^{(E)}\right| \phi_{n}\right\rangle \leq$ 1 which depends only on environmental properties, such as its size $n$ and the initially prepared quantum state $\hat{\rho}^{(E)}$. In particular, three different cases can be distinguished. If all environmental qubits are initially prepared in the eigenstate $|\phi\rangle$ of the interaction $\hat{u}^{\phi}$ of Eq.(2), i.e. $r=1$, the system state $\hat{\rho}^{(S)}$ remains unchanged. If the environmental state $\hat{\rho}^{(E)}$ has zero overlap with quantum 
state $\left|\phi_{n}\right\rangle$, i.e. $r=0$, we observe perfect decoherence in the asymptotic limit $N \gg 1$. In all other cases asymptotic decoherence results in an only partial suppression of coherences between the pointer states of the open system $S$. In particular, if the initial state of the environment is a factorized state $\hat{\xi}^{\otimes n}$, for example, the decoherence factor is given by $r=\left\langle\phi_{1}|\hat{\xi}| \phi_{1}\right\rangle^{n}$. Thus, coherences between pointer state of the system $S$ decrease exponentially with increasing number $n$ of environmental qubits. In this case perfect decoherence can only be obtained for an infinite environment. Furthermore, if environmental qubits share initial correlations even in the case of an environment with infinitely many qubits perfect asymptotic decoherence cannot always be achieved. Let us consider an initially prepared pure environmental state of the form $\hat{\rho}_{e n v}=\left|\chi_{n}\right\rangle\left\langle\chi_{n}\right|$ with $\left|\chi_{n}\right\rangle=\cos \alpha\left|\phi_{n}\right\rangle+\sin \alpha\left|\nu_{n}\right\rangle$ and $\left\langle\phi_{n} \mid \nu_{n}\right\rangle=0$, for example. The associated decoherence factor is given by $r=\cos ^{2}(\alpha)$ and is thus independent of the number of environmental qubits $n$.

Let us now investigate how this decoherence process destroys entanglement in the asymptotic limit of many elementary interactions $N \gg 1$ between system and environment. In order to avoid problems with appropriate measures of entanglement let us focus on an arbitrary two-qubit subsystem of an open quantum system with $k \geq 2$ system qubits and $n$ environmental qubits. Thus, the entanglement of this two-qubit subsystem can be quantified by its concurrence [10].

Consider first of all an initially prepared pure state $\hat{\rho}_{1}^{(S)}=\left|\psi_{1}\right\rangle\left\langle\psi_{1}\right|$ of these two system qubits with $\left|\psi_{1}\right\rangle=$ $a|00\rangle+b|11\rangle$ and $|a|^{2}+|b|^{2}=1$. For an arbitrary environmental state $\hat{\rho}^{(E)}$ the concurrence $C$ of the resulting asymptotic quantum state (7) is given by

$$
C\left(\hat{\rho}_{1 \infty}^{(S)}\right)=2|a b|\left\langle\phi_{n}\left|\hat{\rho}^{(E)}\right| \phi_{n}\right\rangle=C\left(\hat{\rho}_{1}^{(S)}\right) r .
$$

Thus, the resulting asymptotic entanglement is equal to the initial degree of entanglement multiplied by the decoherence factor $r$. Therefore, in the asymptotic limit $N \gg 1$ the disentanglement of the originally prepared entangled state $\hat{\rho}_{1}^{(S)}=\left|\psi_{1}\right\rangle\left\langle\psi_{1}\right|$ is governed by the same dependencies concerning the size $n$ and the initially prepared state of the environment $\hat{\rho}^{(E)}$ as the decoherence factor $r$.

Let us now consider an uncorrelated state of system and environment with the two system qubits initially prepared in the maximally entangled state $\left|\psi_{2}\right\rangle=$ $1 / 2(-|00\rangle+|01\rangle+|10\rangle+|11\rangle)$. For an arbitrary environmental state $\hat{\rho}^{(E)}$ the concurrence $C$ of the resulting asymptotic quantum state (7) is given by

$$
C\left(\hat{\rho}_{2 \infty}^{(S)}\right)=\frac{1}{2} \max \left\{0,3\left\langle\phi_{n}\left|\hat{\rho}^{(E)}\right| \phi_{n}\right\rangle-1\right\} .
$$

Thus, the resulting asymptotic entanglement is drastically different from the previous case. As soon as the decoherence factor decreases below the threshold value of $1 / 3$ the degree of entanglement drops to zero in the asymptotic limit $N \gg 1$. The entanglement of the open quantum system is now very sensitive to the size $n$ and to the state $\hat{\rho}^{(E)}$ of the environment. In particular, consider an $n$-qubit environment initially prepared in a factorized quantum state $\hat{\rho}^{(E)}=\hat{\xi}^{\otimes n}$ with $\left\langle\phi_{n}\left|\hat{\rho}^{(E)}\right| \phi_{n}\right\rangle<1$. This implies the relation $\left\langle\phi_{n}\left|\hat{\rho}^{(E)}\right| \phi_{n}\right\rangle=\langle\phi|\hat{\xi}| \phi\rangle^{n}$. Therefore, as soon as the number $n$ of qubits in the environment exceeds the critical value of

$$
n_{\text {sep }}=\left\lceil-\frac{\ln 3}{\ln \left\langle\phi_{1}|\hat{\xi}| \phi_{1}\right\rangle}\right\rceil,
$$

the asymptotic entanglement of the system state vanishes. ( $\lceil x\rceil$ denotes the largest integer less or equal to $x$.) This is in extreme contrast to the previous case studied in Eq.(8) in which entanglement decreases exponentially with the size $n$ of the environment and never vanishes completely for any finite size of the environment.

The numerical simulations depicted in Fig. 2 2 show the dependence of the concurrence $C$ of the two qubits initially prepared in the quantum state $\left|\psi_{2}\right\rangle$ on the number $N$ of elementary interactions between system and environment. The asymptotic limit is already achieved after a few elementary interactions. In the exceptional case of an environment of minimal size, i.e. $n=1$, Eqs.(4) also allow a solution with eigenvalue $\lambda=-1$ which results in a non-stationary asymptotic limit leading to an oscillatory dependence. In all other cases the asymptotic entanglement is stationary. In the case depicted in Fig. 2 the critical size of the environment is given by $n_{\text {sep }}=4$ so that for all smaller sizes of the environment asymptotically the two-qubit system is not disentangled completely.

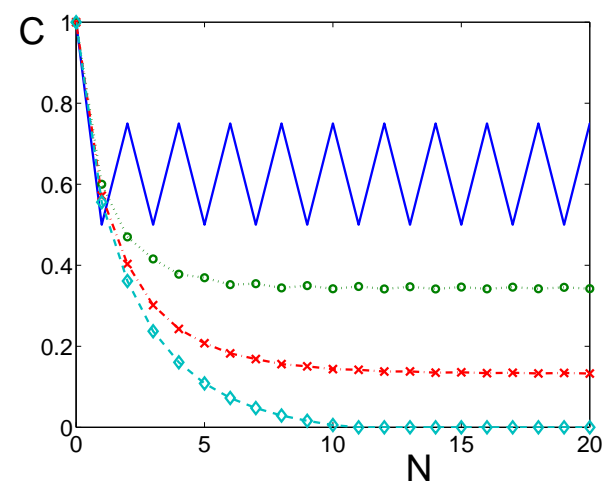

FIG. 2: Dependence of the concurrence $C$ of the two-qubit state $\hat{\rho}^{(S)}(N)$ on the number of elementary interactions $N$ and on the size of the environment $n$ ( $n=1$ full, $n=2$ dotted, $n=3$ dashed-dotted, $n=4$ dashed): The initial state of the environment (system) is given by $|\delta\rangle^{\otimes n}$ with $|\delta\rangle=\sin (\pi / 6)|0\rangle+\cos (\pi / 6)|1\rangle$ $\left(\left|\psi_{2}\right\rangle\right)$ and $\hat{u}^{(\phi)}$ is given by Eq.(2) with $\phi=2 \pi / 3$.

Thus, with respect to asymptotic decoherence-induced disentanglement of an open two-qubit system two classes 
of initially prepared entangled states $\hat{\rho}^{(S)}$ can be distinguished. The first class consists of quantum states which are fragile against decoherence in the sense that their asymptotic entanglement vanishes already for non-zero decoherence factors $r$ (compare with Eq.(9)). In contrast, quantum states $\hat{\rho}^{(S)}$ of the second class are robust against decoherence-induced disentanglement in the sense that the two qubits can be disentangled only if decoherence is perfect, i.e. $r=0$ (compare with Eq.(8) ). As a result these two classes of initially prepared two-qubit states exhibit a very different behavior with respect characteristic features of the decoherence-inducing environment.

Within our general decoherence model even a simple necessary and sufficient condition can be derived whether the entanglement of an initially prepared two-qubit system state $\hat{\rho}^{(S)}$ of an open system is fragile or robust. In view of the general form of the asymptotic system state of Eq.(7) the formal arguments presented in Ref.[11] lead to the conclusions that a two-qubit state $\hat{\rho}^{(S)}$ is fragile with respect to environmental decoherence if and only if its density matrix elements in the pointer basis satisfy the relation

$$
\left\langle 00\left|\hat{\rho}^{(S)}\right| 00\right\rangle\left\langle 10\left|\hat{\rho}^{(S)}\right| 10\right\rangle\left\langle 01\left|\hat{\rho}^{(S)}\right| 01\right\rangle\left\langle 11\left|\hat{\rho}^{(S)}\right| 11\right\rangle \neq 0 .
$$

In summary, a class of decoherence models has been presented by which the intricate interplay between destruction of entanglement and decoherence resulting from the asymptotic long-time interaction of an open qubit system with its environment can be investigated analytically. It does not involve any further simplifying assumptions, such as the ones involved in Markovian models or in models in which each environmental qubit interacts only once with a system qubit. Within this framework it has been shown that two characteristic classes of entangled quantum states can be distinguished, namely fragile and robustly entangled states. They exhibit significantly different dependencies on environmental properties. For two-qubit subsystems of an open qubit system a simple criterion for robustness and fragility has been presented.

The very existence of fragile and robustly entangled states in open quantum systems is a consequence of the characteristic structure of the asymptotic quantum states described by Eq.(9). Without the restriction $0 \leq r \leq 1$ on the decoherence factor a similar structure also appears in other recently discussed decoherence models [1 3,12$]$. Therefore, it can be conjectured that the existence of fragile and robustly entangled quantum states is a general phenomenon accompanying any decoherence process. In view of their significantly weaker sensitivity to decoherence robustly entangled quantum states are expected to play a particularly significant role in the further development of quantum information processing and in its efforts to push entanglement as a characteristic quantum phenomenon as far as possible into the macroscopic domain.

Financial support by the Alexander von Humboldt Foundation, by CASED, and by MSM6840770039 and MSMT LC06002 of the Czech Republic is acknowledged.

[1] E. Joos et al., Decoherence and the Appearance of a Classical World in Quantum Theory, (Springer, Berlin, 2010).

[2] M. A. Schlosshauer, Rev. Mod. Phys. 76, 1267 (2005).

[3] W. H. Zurek, Rev. Mod. Phys. 75, 715 (2003).

[4] T. Yu and J. H. Eberly, Phys. Rev. B 68,165322 (2003).

[5] M. A. Nielsen and I. L. Chuang, Quantum Computation and Quantum Information (Cambridge UP, Cambridge, 2000).

[6] M. Ziman and V. Bužek, Phys. Rev. A 72, 022110 (2005).

[7] J. Novotný, G. Alber, I. Jex, NJP, in print (2011).

[8] I. Bengtsson and K. Życzkowski, Geometry of Quantum States (Cambridge UP, Cambridge, 2006).

[9] J. Novotný, G. Alber, I. Jex, Cent. Eur. J. Phys. 8, 1001 (2010).

[10] S. Hill, W. K. Wootters, Phys. Rev. Lett. 78, 5022 (1997).

[11] J.-H. Huang, S.-Y. Zhu, Phys. Rev. A 76, 062322 (2007).

[12] F. M. Cucchietti, J. P. Paz, W. H. Zurek, Phys. Rev. A 72, 052113 (2005). 